\title{
Editorial: Electrode Materials for Lithium and Post-Lithium Rechargeable Batteries
}

\author{
Ru-Shi Liu ${ }^{1 *}$ and Piercarlo Mustarelli ${ }^{2 *}$ \\ ${ }^{1}$ Department of Chemistry, National Taiwan University, Taipei, Taiwan, ${ }^{2}$ Department of Materials Science, University of Milano \\ Bicocca, Milan, Italy
}

Keywords: batteries and energy storage, lithium, post lithium, anode, cathode

\section{Editorial on the Research Topic}

\section{Electrode Materials for Lithium and Post-Lithium Rechargeable Batteries}

Since their launch in 1991, lithium-ion batteries gave a substantial contribution to change our life paradigms by allowing the development of more powerful and versatile portable electronics. However, their current architecture is not expected to reach the US DOE requirements for

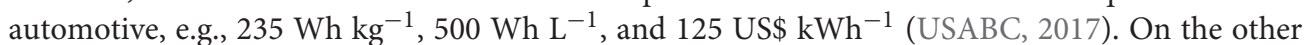
hand, more energy and power density are also needed to fulfill the requirements of grid applications (Goodenough and Park, 2013). Indeed, the substitution of conventional graphite (or Si/C) anode with lithium metal may result in $>50 \%$ increase of energy density and can allow the exploitation of different cathode-reacting species, e.g., oxygen and sulfur (Albertus et al., 2018), leading to new and more performing chemistries, $\mathrm{Li} /$ air and $\mathrm{Li} / \mathrm{S}$, which could deliver theoretical energy density values of the order of 3.5 and $2.5 \mathrm{kWh} \mathrm{kg}^{-1}$, respectively (Bruce et al., 2012).

However, two main challenges prevent lithium metal anodes from full exploitation: (i) safety hazards, and (ii) short cycle life. The safety hazards due to the potential formation of dendrites, eventually short-circuiting the cell, is certainly the most important. Nonetheless, the comparably short cycle life due to the continuous electrolyte decomposition and loss of electrochemically active lithium is also a key performance-limiting factor. The main issues of batteries with a lithium metal anode (LMB) and recent key progresses on methodologies, materials and characterization techniques were recently reported (Lin et al., 2017).

In the frame of the abovementioned scenario, the Research Topic on "Electrode materials for lithium and post-lithium rechargeable batteries" was therefore devoted to the design, modeling, synthesis, and characterization (ex situ, in situ, and operando) of new materials to be employed as anodes or cathodes for lithium and post-lithium (e.g., sodium) rechargeable batteries. The thematic collection was opened to submissions of original papers, mini-reviews, and commentaries that introduce as well as promote a better understanding of emerging materials, methodologies, and new chemistries for advanced energy storage. As expected, the interest of the contributing scientists was completely devoted, both from the research and the reviewing points of view to the investigation of metal ( $\mathrm{Li}, \mathrm{Na}$ ) anode batteries. Several different chemistries were treated, including metal-ion and metal-air.

Delaporte et al. contributed a review paper about the surface pretreatments of lithium foil anode able to control the Solid Electrolyte Interphase (SEI) deposition, and to reduce the severe morphology changes taking place during battery cycling, even in case solid polymer/composite electrolytes, which lead to Li dendrites formation. The authors reviewed recent progress concerning different types of modification able to enhance the electrochemical performance of various battery configurations. Attention was devoted to polymer coatings, carbon coatings, and other inorganic

coatings, as well as to spontaneous chemical reactions with gases and with silane family. Finally, the

Citation:

Liu R-S and Mustarelli P (2020)

Editorial: Electrode Materials for Lithium and Post-Lithium Rechargeable Batteries.

Front. Mater. 7:48.

doi: 10.3389/fmats.2020.00048 
authors carefully considered also mechanical treatments able to reduce lithium surface defects.

Several strategies were recently proposed to suppress dendritic lithium deposition, including: (i) incorporating functional electrolyte additives, (ii) deposition of artificial SEI layers, such as ex-situ formed polymers, and (iii) the exploitation of conductive 3D carbon networks, which serve simultaneously as current collectors and protective layers. This last approach appeared beneficial for the long-term cycling stability of the lithium metal anode, while simultaneously allowing for reduced polarization issues. In this frame, Dou et al. reported an easily-scalable approach for the realization of a free-standing 3D carbon network to support lithium metal deposition, by simply carbonizing filter paper under inert conditions. Lithium plating/stripping experiments reveal highly stable and rather low overpotentials, particularly when ionic liquids were used as the electrolyte.

As previously stated, once the stability problems of the metal anode have been solved, full exploitation of new chemistries, such as metal/air, would be possible. In their contribution, Faktorovich-Simon et al. reported a study on $\mathrm{Na}$ /air cells where the system $\mathrm{Ni}_{\mathrm{x}} \mathrm{Co}_{\mathrm{y}} \mathrm{O}_{\mathrm{z}}$ was employed as a component, with catalytic activity, of the carbon-based cathode. The authors showed that: (i) high-surface-area carbon materials significantly increase the number of

\section{REFERENCES}

Albertus, P., Babinec, S., Litzelman, S., and Newman, A. (2018). Status and challenges in enabling the lithium metal electrode for high-energy and low-cost rechargeable batteries. Nat. Energy 3, 16-21. doi: 10.1038/s41560-017-0047-2

Bruce, P.G., Freunberger, S.A., Hardwick, L.J., and Tarascon, J. M. (2012). Li-O2 and Li-S batteries with high energy storage. Nat. Mater.11, 19-29. doi: $10.1038 /$ nmat3191

Goodenough, J. B., and Park, K.-S. (2013). The Li-Ion rechargeable battery: a perspective. J. Am. Chem. Soc. 135, 1167-1176. doi: 10.1021/ja30 91438

Lin, D., Liu, Y., and Cui, Y. (2017). Reviving the lithium metal anode for high-energy batteries. Nat. Nanotechnol. 12, 194-206. doi: 10.1038/nnano. 2017.16 cyclic- voltammetry runs, without current reduction, and (ii) the best results in terms of electrochemical activity may require a careful balancing of catalytic components, e.g., $45 \% \mathrm{NiCo}_{2} \mathrm{O}_{4}, 10 \% \mathrm{CoO}, 30 \%$ metallic nickel, and $15 \%$ metallic cobalt.

Finally, Wang et al. devoted their attention to lithium-thionyl chloride batteries, which possess one of the highest specific energies among the commercially available electrochemical storage devices. The main limitation of lithium-thionyl chloride batteries is their low discharge capacity at high discharge rates. In Wang et al. study, a porous cathode carbon support was prepared by introducing ammonia bicarbonate as a poreforming agent. The carbonaceous support was then inserted into a commercial battery and enabled the preparation of a high capacity lithium-thionyl chloride battery with a high discharge rate.

Although numerically limited, this Research Topic shows the great vitality of research in the field of rechargeable batteries and offers some interesting ideas for future developments in chemistries based on lithium metal anode.

\section{AUTHOR CONTRIBUTIONS}

All authors listed have made a substantial, direct and intellectual contribution to the work, and approved it for publication.

USABC (2017). USABC Goals for Advanced Batteries for EVs-CY 2020 Commercialization.

Conflict of Interest: The authors declare that the research was conducted in the absence of any commercial or financial relationships that could be construed as a potential conflict of interest.

Copyright (c) 2020 Liu and Mustarelli. This is an open-access article distributed under the terms of the Creative Commons Attribution License (CC BY). The use, distribution or reproduction in other forums is permitted, provided the original author(s) and the copyright owner(s) are credited and that the original publication in this journal is cited, in accordance with accepted academic practice. No use, distribution or reproduction is permitted which does not comply with these terms. 